\author{
Chwan-Huei Tsai \\ Associate Professor, \\ Department of Mechanical Engineering, \\ Huafan University, \\ Taipei Hsien, Taiwan 223, \\ Republic of China \\ Chien-Ching Ma \\ Professor \\ Department of Mechanical Engineering, \\ National Taiwan University, \\ Taipel, Taiwan 10617 \\ Republic of China
}

\title{
Theoretical Transient Analysis of the Interaction Between a Dynamically Propagating In-Plane Crack and Traction-Free Boundaries
}

\begin{abstract}
In this study, the transient response of a propagating in-plane crack interacting with half-plane boundaries is investigated in detail. The reflected waves which are generated from traction-free boundaries will interact with the propagating crack and make the problem extremely difficult to analyze. The complete transient solutions are constructed by superimposing fundamental solutions in the Laplace transform domain. The fundamental solutions represent the responses of applying exponentially distributed loadings in the Laplace transform domain on the surface of a half-plane or the propagating crack faces. We focus our attention on the determination of the dynamic stress intensity factor. The dynamic stress intensity factors of a propagating crack in a configuration with boundaries and subjected to dynamic loadings are obtained in an explicit closed form. The transient solutions obtained in this study are in agreement with the experimental results from the literature. Some interesting phenomena observed in the published experimental works are also identified and discussed. It is concluded that the reflected waves generated from the boundary parallel to the crack have much stronger influence on the propagating crack than those generated from the boundary perpendicular to the crack. When the reflected waves generated from the boundary parallel to the crack return to the moving crack tip, the stress intensity factor will increase rapidly.
\end{abstract}

\section{Introduction}

Most existing analyses of cracked bodies are quasi-static. However, there are numerous situations in which the material inertia becomes significant and must be taken into account. Inertial effects can arise either from applying dynamic loading to a cracked solid or from rapid crack propagation. The inherent time dependence of a dynamic fracture process results in mathematical models that are more complex than equivalent quasistatic models. The phenomena of crack propagation, arrest, and branching are very important subjects in the area of dynamic fracture analysis. In a series of papers, Freund (1972a, b, 1973, 1974) developed an important analytical method for evaluating the transient stress field and dynamic stress intensity factor of a propagating semi-infinite crack in an infinite medium. Freund proposed a superposition method in the time domain to study the dynamic effect of crack propagation, some fundamental solutions were obtained and were used to develop the solution for the negation of the stress distribution on the prospective fracture plane. His method is valid for the loading condition of step stress pulse applied on the crack faces, but without considering the reflected waves generated from boundaries.

The interaction of reflected waves with a moving crack has only been discussed in a series of experimental works by RaviChandar and Knauss (1982, 1984a, b, c, d), Ravichandran and

\footnotetext{
Contributed by the Applied Mechanics Division of The AMERICAN SOCIETY of Mechanical EnginesRs for publication in the ASME Journal of Applied MeChanics.

Discussion on this paper should be addressed to the Technical Editor, Professor Lewis T. Wheeler, Department of Mechanical Engineering, University of Houston, Houston, TX 77204-4792, and will be accepted until four months after final publication of the paper itself in the ASME JourNaL OF APPLIED MECHANICS.

Manuscript received by the ASME Applied Mechanics Division, July 26, 1996; final revision, Mar. 14, 1997. Associate Technical Editor: W. J. Drugan.
}

Clifton (1989), and Kim (1985). Experimental results indicated that the effect of reflected waves dominates the stability of crack propagation. The possibility of arrest, acceleration, or branching of the propagating crack is influenced by the reflected waves generated from boundaries of specimen. It seems difficult to obtain the analytical solution of the problem of interaction of reflected waves with a propagating crack by using conventional methodology.

In recent years, a powerful and efficient methodology based on superimposing the fundamental solution in the Laplace transform domain was proposed by Tsai and Ma (1991, 1992, 1997) to solve many complicated and difficult problems. Using this superposition methodology, $\mathrm{Ma}$ and Chen (1994) and $\mathrm{Ma}$ and Ing (1995) investigated the transient response of antiplane semiinfinite cracks in a configuration with boundaries. The solutions to applying an exponentially distributed traction at the surface of a half-plane and at a semi-infinite crack face in the Laplace transform domain are considered as the fundamental solutions. The reflected and diffracted fields generated from the half-plane and crack tip can be obtained by superimposing these fundamental solutions as shown in the Appendix. In this study, the superposition method that is used to solve the stationary crack problem is extended to solve the transient response of a propagating crack interacting with reflected waves generated from tractionfree boundaries.

In this paper, transient stress intensity factors for a semiinfinite crack embedded in a half-plane subjected to a sudden impact on the crack faces are analyzed. The orientations of the half-plane boundary are parallel to the crack and perpendicular to the crack. The reflected waves will induce mixed-mode stress intensity factors and influence the crack propagation path for the crack which is parallel to the boundary. The numerical results obtained in this study show that reflected waves gener- 


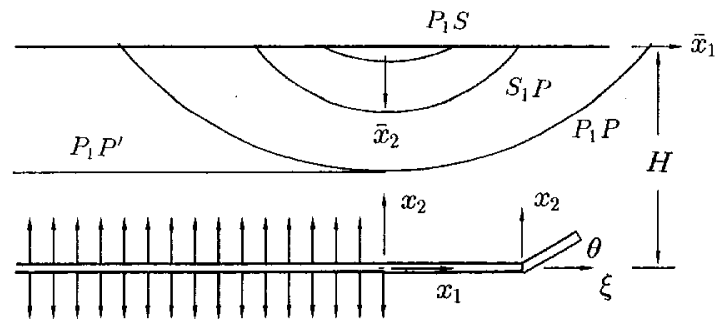

Fig. 1 Configuration and coordinate systems of a semi-infinite crack which is parallel to the half-plane boundary

ated from the boundary parallel to the crack have much stronger influence on the propagating crack than those generated from the boundary perpendicular to the crack. When the reflected waves generated from the boundary parallel to the crack return to the moving crack tip, the stress intensity factor will increase rapidly. Finally, the analytical solution of a propagating crack in a rectangular finite plate subjected to impact loading on the crack faces is obtained without considering the diffracted waves generated from plate corners. The experimental results obtained by Ravi-Chandar and Knauss $(1982,1984 \mathrm{~b})$ are discussed and are compared with the analytical result in light of the theoretical analysis in this study.

\section{Crack Propagation and Interaction With a Half- Plane}

For the convenience of the following derivation, some useful symbols are defined as follows:

$$
\begin{aligned}
& \alpha_{ \pm}=\alpha_{ \pm}(\lambda)=[a \pm \lambda(1 \mp a v)]^{1 / 2}, \\
& \alpha_{ \pm}^{0}=\alpha_{ \pm}^{0}(\lambda)=(a \mp \lambda)^{1 / 2} \text {, } \\
& \alpha=\alpha(\lambda)=\alpha_{+}(\lambda) \alpha_{-}(\lambda), \quad \alpha^{0}=\alpha^{0}(\lambda)=\alpha_{+}^{0}(\lambda) \alpha_{-}^{0}(\lambda), \\
& \beta_{ \pm}=\beta_{ \pm}(\lambda)=[b \pm \lambda(1 \mp b v)]^{1 / 2}, \\
& \beta_{ \pm}^{0}=\beta_{ \pm}^{0}(\lambda)=(b \mp \lambda)^{1 / 2}, \\
& \beta=\beta(\lambda)=\beta_{+}(\lambda) \beta_{-}(\lambda), \quad \beta^{0}=\beta^{0}(\lambda)=\beta_{+}^{0}(\lambda) \beta_{-}^{0}(\lambda), \\
& R=R(\lambda)=\left(b^{2}(1-\lambda v)^{2}-2 \lambda^{2}\right)^{2}+4 \lambda^{2} \alpha \beta \\
& =\kappa(d-\lambda)^{2}\left(c_{1}-\lambda\right)\left(c_{2}+\lambda\right) S_{+}(\lambda) S_{-}(\lambda), \\
& \kappa=4\left(1-a^{2} v^{2}\right)^{1 / 2}\left(1-b^{2} v^{2}\right)^{1 / 2}-\left(2-b^{2} v^{2}\right)^{2}, \quad d=1 / v, \\
& a_{1}=a /(1+a v), \quad b_{1}=b /(1+b v), \quad c_{1}=c /(1+c v), \\
& a_{2}=a /(1-a v), \quad b_{2}=b /(1-b v), \quad c_{2}=c /(1-c v), \\
& S_{ \pm}=S_{ \pm}(\lambda) \\
& =\exp \left(\frac{-1}{\pi} \int_{a_{2,1}}^{b_{2,1}} \tan ^{-1}\left[\frac{4 \xi^{2}|\alpha \| \beta|}{\left(b^{2}(1 \pm \xi \nu)^{2}-2 \xi^{2}\right)^{2}}\right] \frac{d \xi}{\xi \pm \lambda}\right), \\
& R^{0}=R^{0}(\lambda)=\left.R(\lambda)\right|_{v=0}, \quad S_{ \pm}^{0}=S_{ \pm}^{0}(\lambda)=\left.S_{ \pm}(\lambda)\right|_{\nu=0},
\end{aligned}
$$

where $a\left(=1 / v_{l}\right), b\left(=1 / v_{s}\right), c\left(=1 / v_{r}\right)$, and $d(=1 / v)$ are the slownesses of longitudinal wave, shear wave, Rayleigh wave, and moving crack tip, respectively, $v_{l}, v_{s}, v_{r}$, and $v$ are the corresponding speeds of the longitudinal wave, shear wave, Rayleigh wave, and the moving crack tip.

We consider a semi-infinite crack embedded in a half-plane whose boundary is parallel to the crack as shown in Fig. 1 and embedded in a half-plane whose boundary is perpendicular to the crack as shown in Fig. 2. The distance from the crack tip to the boundary parallel to the crack is denoted as $H$ and to the boundary perpendicular to the crack is denoted as $L$. The coordinate system $\left(x_{1}, x_{2}\right)$ is attached to the stationary crack tip, and the alternative coordinate system $\left(\bar{x}_{1}, \bar{x}_{2}\right)$ is fixed at $x_{1}$ $=0, x_{2}=H\left(\right.$ or $\left.x_{1}=L, x_{2}=0\right)$. At time $t=0$, a uniform pressure of magnitude $\sigma_{0}$ is suddenly applied to the crack faces. After a delay time $t_{d}$, the crack begins to extend at a constant velocity $v$, and the moving coordinate system $\left(\xi, x_{2}\right)$ is attached to the moving crack tip. The relation between $x_{1}$ and $\xi$ is $x_{1}=$ $\xi+v\left(t-t_{d}\right)$.

After a uniformly distributed impact is suddenly applied to the stationary crack faces, a diffracted longitudinal wave (denoted by $P_{1}$ ) and a shear wave (denoted by $S_{1}$ ) will be emitted from the stationary crack tip. The $P_{1}$ wave will be reflected from the traction-free boundary of the half-plane and hence generate the reflected longitudinal wave and shear wave which are denoted by $P_{1} P$ wave and $P_{1} S$ wave. Similarly, the $S_{1}$ wave will be reflected from the boundary of the half-plane and the reflected longitudinal wave $\left(S_{1} P\right)$ and shear wave $\left(S_{1} S\right)$ will be generated. After a delay time $t_{d}$, the crack begins to propagate with a constant speed $v$. The diffracted longitudinal wave $\left(P_{2}\right)$ and shear wave $\left(S_{2}\right)$ will be emitted from the moving crack tip at time $t=t_{t}$. The diffracted waves $P_{2}$ and $S_{2}$ will also be reflected from the boundary of the half-plane and generate reflected longitudinal waves $\left(P_{2} P, S_{2} P\right)$ and shear waves $\left(P_{2} S\right.$, $S_{2} S$ ). All these reflected waves will interact with the moving crack tip at later times, and make the problem extremely difficult to analyze.

Before reflected waves return to the propagating crack tip, the problem can be viewed as a semi-infinite crack propagating in an unbounded medium; the solution has been obtained by Freund (1973) by using another method. We focus our attention on the reflected waves for the first time. The reflected effect for the second time, third time, etc., will not be discussed in this study.

The diffracted waves include two parts, the first one is induced from the stationary crack tip and the second one is generated from the moving crack tip. Before the stress waves which are emitted from the propagating crack tip return to the moving crack tip, the stress intensity factors are shown in Section 2.1. After the $P_{2} P, P_{2} S, S_{2} P$, and $S_{2} S$ waves reach the crack tip, the stress intensity factors can be obtained approximately by taking the problem for a uniformly extending crack (i.e., At time $t=0$, the crack tip is located at $x_{1}=-v t_{d}$ and extends at a constant speed $v$ without delay time) which is shown in Section 2.2 .

\subsection{Stress Intensity Factors due to the Stress Waves Generated From the Stationary Crack.}

2.1.1 Diffracted Waves in Infinite Medium. Consider a stationary semi-infinite crack embedded in a half-plane as

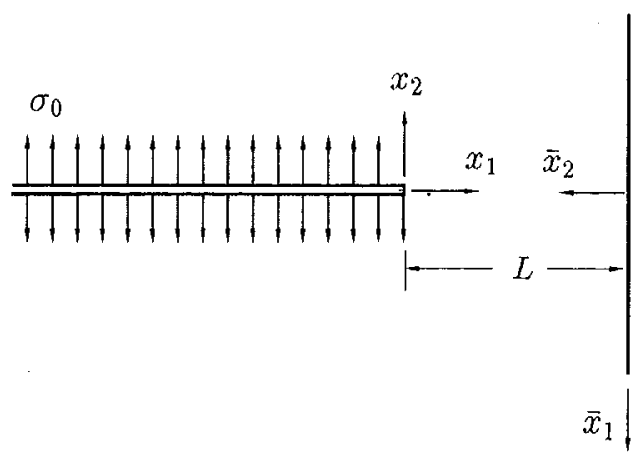

Fig. 2 Configuration and coordinate systems of a semi-infinite crack which is perpendicular to the half-plane boundary 
shown in Fig. 1. At time $t=0$, a uniform pressure with magnitude $\sigma_{0}$ is suddenly applied at the crack faces. The waves emitted from the stationary crack can be expressed in the Laplace transform domain as follows (Thau and $\mathrm{Lu}, 1971$ ):

$$
\begin{aligned}
\bar{\sigma}_{22}^{D}=\frac{A_{0}}{2 \pi i p} \int \frac{\left(b^{2}-2 \lambda^{2}\right)^{2}}{2 \lambda \alpha_{+}^{0}(c-\lambda) S_{-}^{0}} e^{-p \alpha^{0} x_{2}+p \lambda x_{1}} \\
+\frac{2 \lambda \alpha_{-}^{0} \beta^{0}}{(c-\lambda) S_{-}^{0}} e^{-p \beta^{0} x_{2}+p \lambda x_{1}} d \lambda,
\end{aligned}
$$

$\bar{\sigma}_{12}^{D}=\frac{A_{0}}{2 \pi i p} \int \frac{-\alpha_{-}^{0}\left(b^{2}-2 \lambda^{2}\right)}{(c-\lambda) S_{-}^{0}} e^{-p \alpha^{0} x_{2}+p \lambda \lambda x_{1}}$

$$
+\frac{\alpha_{-}^{0}\left(b^{2}-2 \lambda^{2}\right)}{(c-\lambda) S_{-}^{0}} e^{-p \beta^{0} x_{2}+p \lambda x_{1}} d \lambda
$$

where

$$
A_{0}=\frac{\omega_{0}}{\left(b^{2}-a^{2}\right)}, \quad \omega_{0}=\frac{\sigma_{0} \sqrt{a}}{c S_{+}^{0}(0)},
$$

$p$ is the Laplace transform parameter. The first and second terms of (2) and (3) represent the diffracted $P_{1}$ wave and $S_{1}$ wave generated from the stationary crack tip, respectively. The solution is exactly the same as that for a semi-infinite crack in an unbounded medium. The corresponding stress intensity factor in the time domain is

$$
K_{I}=\frac{2 \sqrt{2}}{\sqrt{\pi}} \omega_{0} \sqrt{t}
$$

2.1.2 Stress Intensity Factors due to Reflected Waves From the Boundary Parallel to the Crack. The diffracted waves emitted from the stationary crack will be reflected from the boundary at $x_{2}=H$. From Eqs. (2) and (3), it is obvious that the traction which must be applied at $x_{2}=H$ to eliminate the stresses $\bar{\sigma}_{22}^{D}$ and $\bar{\sigma}_{12}^{D}$ is represented by the exponential function $e^{p \lambda x_{1}}$. The fundamental solutions for normal traction $e^{p \lambda x_{1}}$ and tangential traction $e^{p \lambda x_{1}}$ applied at the surface of the half-plane can be obtained from the Appendix by taking $v \rightarrow 0, \xi \rightarrow \bar{x}_{1}, \eta$ $\rightarrow \lambda$. Then the stresses $\bar{\sigma}_{22}^{R}$ and $\bar{\sigma}_{12}^{R}$ induced by reflected waves can be obtained by superimposing the fundamental solutions of (A4), (A5), (A9), and (A10).

If the crack starts to propagate before the reflected wave $P_{1} P$ returns to the crack tip (i.e., $t_{d}<2 a H$ ), the moving coordinate system $\left(\xi, x_{2}\right)$ should be introduced to perform the superposition procedure for the propagating crack. The stresses induced on the propagating crack faces by $\bar{\sigma}_{22}^{R}$ and $\vec{\sigma}_{12}^{R}$ must be eliminated. Applying the transformation principle between the two different coordinate systems as mentioned in Appendix Eqs. (A22) and (A23), $\bar{\sigma}_{22}^{R}$ and $\bar{\sigma}_{12}^{R}$ can be obtained as

$$
\begin{aligned}
& \bar{\sigma}_{22}^{R}= \frac{A_{0}}{2 \pi i p} \int \frac{-\left(b^{2}-2 \bar{\lambda}^{2}\right)^{2}\left(\left(b^{2}-2 \bar{\lambda}^{2}\right)^{2}-4 \bar{\lambda}^{2} \bar{\alpha}^{0} \bar{\beta}^{0}\right)}{2 \bar{\lambda} \bar{\alpha}_{+}^{0}(c-\bar{\lambda}) \bar{S}_{-}^{0} \bar{R}^{0}(1-\lambda v)^{2}} \\
& \times e^{-2 p \alpha H+p \lambda\left(\xi-v t_{d}\right)} d \lambda \\
&+\frac{A_{0}}{2 \pi i p} \int \frac{-8 \bar{\lambda} \bar{\alpha}^{0} \bar{\beta}^{0}\left(b^{2}-2 \bar{\lambda}^{2}\right)^{2}}{\bar{\alpha}_{+}^{0}(c-\bar{\lambda}) \bar{S}_{-}^{0} \bar{R}^{0}(1-\lambda v)^{2}} \\
& \times e^{-p \alpha H-p \beta H+p \lambda\left(\xi-v t_{d}\right)} d \lambda \\
&+\frac{A_{0}}{2 \pi i p} \int \frac{2 \bar{\lambda} \bar{\alpha}_{-}^{0} \bar{\beta}^{0}\left(\left(b^{2}-2 \bar{\lambda}^{2}\right)^{2}-4 \bar{\lambda}^{2} \bar{\alpha}^{0} \bar{\beta}^{0}\right)}{(c-\bar{\lambda}) \bar{S}_{-}^{0} \bar{R}^{0}(1-\lambda v)^{2}} \\
& \bar{\sigma}_{12}^{\prime \prime}= \frac{A_{0}}{2 \pi i p} \int \frac{-\bar{\alpha}_{-}^{0}\left(b^{2}-2 \bar{\lambda}^{2}\right)\left(\left(b^{2}-2 \bar{\lambda}^{2}\right)^{2}-4 \bar{\lambda}^{2} \bar{\alpha}^{0} \bar{\beta}^{0}\right)}{(c-\bar{\lambda}) \bar{S}_{-}^{0} \bar{R}^{0}(1-\lambda v)^{2}} \\
& \times e^{-2 p \alpha H+p \lambda\left(\xi-v t_{d}\right)} d \lambda \\
&+\frac{A_{0}}{2 \pi i p} \int \frac{2 \bar{\alpha}_{-}^{0}\left(b^{2}-2 \bar{\lambda}^{2}\right)\left(\left(b^{2}-2 \bar{\lambda}^{2}\right)^{2}-4 \bar{\lambda}^{2} \bar{\alpha}^{0} \bar{\beta}^{0}\right)}{(c-\bar{\lambda}) \bar{S}_{-}^{0} \bar{R}^{0}(1-\lambda v)^{2}} \\
& \times e^{-p \alpha H-p \beta H+p \lambda\left(\xi-v t_{d}\right) d \lambda} \\
&+\frac{A_{0}}{2 \pi i p} \int \frac{-\bar{\alpha}_{--}^{0}\left(b^{2}-2 \bar{\lambda}^{2}\right)\left(\left(b^{2}-2 \bar{\lambda}^{2}\right)^{2}-4 \bar{\lambda}^{2} \bar{\alpha}^{0} \bar{\beta}^{0}\right)}{(c-\bar{\lambda}) \bar{S}_{-}^{0} \bar{R}^{0}(1-\lambda v)^{2}} \\
& \times e^{-2 p \beta H+p \lambda\left(\xi-v t_{d}\right)} d \lambda
\end{aligned}
$$

where

$$
\bar{\lambda}=\frac{\lambda}{1-\lambda v}, \quad \bar{\alpha}^{0}=\alpha^{0}(\bar{\lambda}), \quad \bar{\alpha}_{ \pm}^{0}=\alpha_{ \pm}^{0}(\bar{\lambda}),
$$

$\bar{\beta}^{0}=\beta^{0}(\bar{\lambda}), \quad \bar{\beta}_{ \pm}^{0}=\beta_{ \pm}^{0}(\bar{\lambda}), \quad \bar{S}^{0}=S^{0}(\bar{\lambda}), \quad \bar{R}^{0}=R^{0}(\bar{\lambda})$.

It is obvious that the tractions which should be applied to the propagating crack faces to eliminate the stresses $\bar{\sigma}_{22}^{R}$ and $\bar{\sigma}_{12}^{R}$ induced by reflected waves are represented by the function $e^{p \lambda \xi}$. Since the stress intensity factor for applying normal traction $e^{m \eta \xi}$ at the propagating crack faces has been obtained in (A16) and (A21), the stress intensity factors induced from reflected waves can be obtained by superimposing the fundamental solution as follows:

$$
\begin{aligned}
\bar{K}_{I}= & \frac{1}{2 \pi i p^{3 / 2}} \int H_{1}(\lambda) e^{-2 p \alpha H-p \lambda \nu t_{d}} \\
& \quad+H_{2}(\lambda) e^{-p \alpha H-p \beta H-p \lambda \nu t_{d}}+H_{3}(\lambda) e^{-2 p \beta H-p \lambda u t_{d}} d \lambda, \\
\bar{K}_{I I}= & \frac{1}{2 \pi i p^{3 / 2}} \int H_{4}(\lambda) e^{-2 p \alpha H-p \lambda u u_{d}} \\
& \quad+H_{5}(\lambda) e^{-p \alpha H-p \beta H-p \lambda u_{d}}+H_{6}(\lambda) e^{-2 p \beta H-p \lambda v t_{d}} d \lambda,
\end{aligned}
$$

where

$$
\begin{gathered}
H_{1}(\lambda)=-\left(b^{2}-2 \bar{\lambda}^{2}\right)^{2}\left(\left(b^{2}-2 \bar{\lambda}^{2}\right)^{2}\right. \\
\left.\quad-4 \bar{\lambda}^{2} \bar{\alpha}^{0} \bar{\beta}^{0}\right) \alpha_{+} J(\lambda) / 2 \sqrt{1-a v} \bar{\lambda} \bar{\alpha}_{+}^{0}, \\
H_{2}(\lambda)=-8 \bar{\lambda} \bar{\alpha}_{-}^{0} \bar{\beta}^{0}\left(b^{2}-2 \bar{\lambda}^{2}\right)^{2} \alpha_{+} J(\lambda) / \sqrt{1-a v}, \\
H_{3}(\lambda)=2 \bar{\lambda} \bar{\alpha}_{-}^{0} \bar{\beta}^{0}\left(\left(b^{2}-2 \bar{\lambda}^{2}\right)^{2}-4 \bar{\lambda}^{2} \bar{\alpha}^{0} \bar{\beta}^{0}\right) \alpha_{+} J(\lambda) / \sqrt{1-a v}, \\
H_{4}(\lambda)=-\bar{\alpha}_{-}^{0}\left(b^{2}-2 \bar{\lambda}^{2}\right)\left(\left(b^{2}-2 \bar{\lambda}^{2}\right)^{2}\right. \\
\left.-4 \bar{\lambda}^{2} \bar{\alpha}^{0} \bar{\beta}^{0}\right) \beta_{+} J(\lambda) / \sqrt{1-b v,} \\
H_{5}(\lambda)=-2 H_{4}(\lambda), \quad H_{6}(\lambda)=H_{4}(\lambda), \\
J(\lambda)=\sqrt{2} A_{0} /\left((c-\bar{\lambda}) \bar{S}_{-}^{0} \bar{R}^{0}(1-\lambda v)^{2}\left(c_{2}+\lambda\right) S_{+}\right) .
\end{gathered}
$$

Inverting to the time domain by using the Cagniard-de Hoop method, the stress intensity factors of the propagating crack induced by reflected $P_{1} P, P_{1} S, S_{1} P$, and $S_{1} S$ waves can be obtained as follows:

$$
\begin{aligned}
K_{I}= & \frac{1}{\pi^{3 / 2}} \int_{T_{P_{1} P}}^{t} \sqrt{t-\tau} \operatorname{Im}\left[H_{1}\left(\lambda_{1}\right) \frac{\partial \lambda_{1}}{\partial \tau}\right] d \tau \\
& +\frac{1}{\pi^{3 / 2}} \int_{T_{P_{1} S}}^{t} \sqrt{t-\tau} \operatorname{Im}\left[H_{2}\left(\lambda_{2}\right) \frac{\partial \lambda_{2}}{\partial \tau}\right] d \tau \\
& +\frac{1}{\pi^{3 / 2}} \int_{T_{S_{1} S}}^{t} \sqrt{t-\tau} \operatorname{Im}\left[H_{3}\left(\lambda_{3}\right) \frac{\partial \lambda_{3}}{\partial \tau}\right] d \tau \\
K_{I I}= & \frac{1}{\pi^{3 / 2}} \int_{T_{P_{1} P}}^{t} \sqrt{t-\tau} \operatorname{Im}\left[H_{4}\left(\lambda_{1}\right) \frac{\partial \lambda_{1}}{\partial \tau}\right] d \tau \\
& +\frac{1}{\pi^{3 / 2}} \int_{T_{P_{1} S}}^{t} \sqrt{t-\tau} \operatorname{Im}\left[H_{5}\left(\lambda_{2}\right) \frac{\partial \lambda_{2}}{\partial \tau}\right] d \tau \\
& +\frac{1}{\pi^{3 / 2}} \int_{T_{S_{1} S}}^{t} \sqrt{t-\tau} \operatorname{Im}\left[H_{6}\left(\lambda_{3}\right) \frac{\partial \lambda_{3}}{\partial \tau}\right] d \tau
\end{aligned}
$$

where

$$
\lambda_{1}=\frac{v t_{d} t-4 v a^{2} H^{2}+i 2 H \sqrt{t^{2}-a^{2}\left(v t-v t_{d}\right)^{2}-4 a^{2} H^{2}}}{v^{2} t_{d}^{2}+4 H^{2}-4 a^{2} v^{2} H^{2}},
$$




$$
\lambda_{3}=\frac{v t_{d} t-4 v b^{2} H^{2}+i 2 H \sqrt{t^{2}-b^{2}\left(v t-v t_{d}\right)^{2}-4 b^{2} H^{2}}}{v^{2} t_{d}^{2}+4 H^{2}-4 b^{2} v^{2} H^{2}},
$$

and $\lambda_{2}$ is the solution of $\alpha H+\beta H+\lambda v t_{d}=t$. The arrival time for each wavefront is

$$
\begin{aligned}
& T_{P_{1} P}=\frac{-a^{2} v^{2} t_{d}+\sqrt{a^{2} v^{2} t_{d}^{2}+4 a^{2} H^{2}-4 a^{4} v^{2} H^{2}}}{1-a^{2} v^{2}}, \\
& T_{S_{1} S}=\frac{-b^{2} v^{2} t_{d}+\sqrt{b^{2} v^{2} t_{d}^{2}+4 b^{2} H^{2}-4 b^{4} v^{2} H^{2}}}{1-b^{2} v^{2}},
\end{aligned}
$$

and $T_{P_{1} S}$ is the corresponding time $t$ at which the imaginary part of $\lambda_{2}$ begins to vanish.

The physical meaning of the terms in (9) and (10) following the sequence represent the stress intensity factors due to the
2.1.3 Stress Intensity Factors due to Reflected Waves From the Boundary Perpendicular to the Crack. If the crack is perpendicular to the half-plane boundary as shown in Fig. 2, the diffracted waves emitted from the stationary crack tip will be reflected from the boundary at $x_{1}=L$. It is obvious that the traction which must be applied at $x_{1}=$ $L$ to eliminate the stresses $\bar{\sigma}_{11}^{D}$ and $\bar{\sigma}_{12}^{D}$ can be represented by the exponential functions $e^{-p x^{0}\left|F_{1}\right|}, e^{-p \beta^{0}\left|F_{1}\right|}$. The fundamental solutions for applying normal traction and tangential traction $e^{-p \alpha^{0}\left|x_{1}\right|}$ or $e^{-p \beta^{\gamma}\left|\bar{x}_{1}\right|}$ to the surface of a half-plane were solved by Tsai and Ma (1991); the reflected waves can be obtained by superimposing these fundamental solutions. Then, by using the same procedure as given from (5) to (12), the stress intensity factor of the propagating crack induced by reflected waves from the boundary of half-plane can be expressed as follows:

$$
\begin{aligned}
K_{l}(t)= & \frac{\sqrt{2} A_{0}}{\pi^{3 / 2}} \int_{T_{P_{1}, p}}^{t} \sqrt{t-\tau} \operatorname{Im}\left[\frac{\left(b^{2}-2 \bar{\lambda}_{1}^{2}\right)^{2}\left(\left(b^{2}-2 \bar{\alpha}^{0^{2}}\right)^{2}-4 \bar{\lambda}_{1} \bar{\alpha}^{0^{2}} \beta^{0}\left(\bar{\alpha}^{0}\right)\right) \alpha_{+}\left(\lambda_{1}\right)}{2 \bar{\lambda}_{1} \bar{\alpha}_{-}^{0}\left(c+\bar{\lambda}_{1}\right) S_{+}^{0}\left(\bar{\lambda}_{1}\right) R^{0}\left(\bar{\alpha}^{0}\right)\left(1-\lambda_{1} v\right) \sqrt{1-a v}\left(c_{2}+\lambda_{1}\right) S_{+}\left(\lambda_{1}\right)} \frac{\partial \lambda_{1}}{\partial \tau}\right] d \tau \\
& +\frac{2 \sqrt{2} A_{0}}{\pi^{3 / 2}} \int_{T_{P_{1} S}}^{t} \sqrt{t-\tau} \operatorname{Im}\left[\frac{-4\left(b^{2}-2 \alpha^{02}\left(\bar{\beta}^{0}\right)\right)\left(\alpha_{+}^{0}\left(\alpha^{0}\left(\bar{\beta}^{0}\right)\right) \bar{\beta}^{0} \bar{\lambda}_{2}^{2} \alpha_{+}\left(\lambda_{2}\right)\right.}{\alpha^{0}\left(\bar{\beta}^{0}\right)\left(c+\alpha^{0}\left(\bar{\beta}^{0}\right)\right) S_{+}^{0}\left(\alpha^{0}\left(\bar{\beta}^{0}\right)\right)\left(1-\lambda_{2} v\right) \sqrt{1-a v}\left(c_{2}+\lambda_{2}\right) S_{+}\left(\lambda_{2}\right)} \frac{\partial \lambda_{2}}{\partial \tau}\right] d \tau \\
& +\frac{2 \sqrt{2} A_{0}}{\pi^{3 / 2}} \int_{T_{S_{1} P}}^{t} \sqrt{t-\tau} \operatorname{Im}\left[\frac{-4\left(b^{2}-2 \bar{\lambda}_{3}^{2}\right)\left(b^{2}-2 \bar{\alpha}^{0^{2}}\right) \alpha_{+}^{0}\left(\beta^{0}\left(\bar{\alpha}^{0}\right)\right) \bar{\alpha}^{0} \bar{\lambda}_{3} \alpha_{+}\left(\lambda_{3}\right)}{\left(c+\beta^{0}\left(\bar{\alpha}^{0}\right)\right) S_{+}^{0}\left(\beta^{0}\left(\bar{\alpha}^{0}\right)\right) R^{0}\left(\bar{\alpha}^{0}\right)\left(1-\lambda_{3} v\right) \sqrt{1-a v}\left(c_{2}+\lambda_{3}\right) S_{+}\left(\lambda_{3}\right)} \frac{\partial \lambda_{3}}{\partial \tau}\right] d \tau \\
& +\frac{2 \sqrt{2} A_{0}}{\pi^{3 / 2}} \int_{T_{S_{1} S}} \sqrt{t-\tau} \operatorname{Im}\left[\frac{\left(-2 \bar{\alpha}_{+}^{0} \bar{\beta}^{0} \bar{\lambda}_{4}\left(b^{2}-2 \bar{\lambda}^{2}\right)^{2}+8 \bar{\alpha}_{+}^{0} \bar{\beta}^{0^{3}} \bar{\lambda}_{4}^{2} \alpha^{0}\left(\bar{\beta}^{0}\right)\right) \alpha_{+}\left(\lambda_{4}\right)}{\left(c+\bar{\lambda}_{4}\right) S_{+}^{0}\left(\bar{\lambda}_{4}\right) R^{0}\left(\bar{\beta}^{0}\right)\left(1-\lambda_{4} v\right) \sqrt{1-a v}\left(c_{2}+\lambda_{4}\right) S_{+}\left(\lambda_{4}\right)} \frac{\partial \lambda_{4}}{\partial \tau}\right] d \tau,
\end{aligned}
$$

where

$$
\begin{aligned}
\lambda_{1}= & \frac{\tau}{2 L+v t_{d}}, \quad \lambda_{4}=\frac{\tau}{2 L+v t_{d}}, \\
\lambda_{2}= & \frac{a^{2} v L^{2}-b^{2} v L^{2}-\tau L-\tau v t_{d}}{a^{2} v^{2} L^{2}-b^{2} v^{2} L^{2}-v^{2} t_{d}^{2}-2 L v t_{d}} \\
& +\frac{\left[\left(a^{2} v L^{2}-b^{2} v L^{2}-\tau L-\tau v t_{d}\right)^{2}-\left(a^{2} v^{2} L^{2}-b^{2} v^{2} L^{2}-v^{2} t_{d}^{2}-2 L v t_{d}\right)\left(a^{2} L^{2}-b^{2} L^{2}-\tau^{2}\right)\right]^{1 / 2}}{a^{2} v^{2} L^{2}-b^{2} v^{2} L^{2}-v^{2} t_{d}^{2}-2 L v t_{d}}, \\
\lambda_{3}= & \frac{b^{2} v L^{2}-a^{2} v L^{2}-\tau L-\tau v t_{d}}{b^{2} v^{2} L^{2}-a^{2} v^{2} L^{2}-v^{2} t_{d}^{2}-2 L v t_{d}} \\
& +\frac{\left[\left(b^{2} v L^{2}-a^{2} v L^{2}-\tau L-\tau v t_{d}\right)^{2}-\left(b^{2} v^{2} L^{2}-a^{2} v^{2} L^{2}-v^{2} t_{d}^{2}-2 L v t_{d}\right)\left(b^{2} L^{2}-a^{2} L^{2}-\tau^{2}\right)\right]^{1 / 2}}{b^{2} v^{2} L^{2}-a^{2} v^{2} L^{2}-v^{2} t_{d}^{2}-2 L v t_{d}} .
\end{aligned}
$$

contribution of reflected $P_{1} P, P_{1} S+S_{1} P$, and $S_{1} S$ waves, respectively. It is noted that the $P_{1} S$ wave and $S_{1} P$ wave reach the propagating crack tip at the same time. The wave fronts of the above-mentioned reflected waves are shown in Fig. 1.

If the crack remains stationary after the reflected waves return to the crack tip, $\lambda_{1}, \lambda_{2}$, and $\lambda_{3}$ will be all pure imaginary numbers. Then, the integral result of (9) comes only from the contributions of the pole $\lambda_{1}=0$ of the first term, and the stress intensity factor can be reduced to the simple form

$$
K_{I}=\frac{2 \sqrt{2} \omega_{0}}{\sqrt{\pi}} \sqrt{t-2 H a} .
$$

Combining the incident effect due to diffracted $P_{1}$ and $S_{1}$ waves, the stress intensity factor is

$$
K_{I}=\frac{2 \sqrt{2} \omega_{0}}{\sqrt{\pi}} \sqrt{t}+\frac{2 \sqrt{2} \omega_{0}}{\sqrt{\pi}} \sqrt{t-2 H a}, \quad 2 H a<t<4 H a .
$$

The arrival time of each wavefront is

$$
\begin{aligned}
& T_{P_{1} P}=a_{1}\left(2 L+v t_{d}\right), \quad T_{P_{\mathrm{l}} S}=\frac{a L+b L+b v t_{d}}{1+b v}, \\
& T_{S_{1} P}=\frac{a L+b L+a v t_{d}}{1+a v}, \quad T_{S_{1} S}=b_{1}\left(2 L+v t_{d}\right) .
\end{aligned}
$$

The physical meanings of the four terms in (15) are the induced dynamic stress intensity factors due to the reflected $P_{1} P, P_{1} S$, $S_{1} P$, and $S_{1} S$ waves generated from the vertical boundary.

\subsection{Stress Intensity Factors due to Stress Waves From the Propagating Crack.}

2.2.1 Diffracted Waves in Infinite Medium. Consider a semi-infinite crack embedded in a half-plane as shown in Fig. 1. At time $t=0$, the crack tip is located at $x_{1}=-v t_{d}$ and extends at a constant speed $v$. At this instant, a dynamic uniform pressure with magnitude $\sigma_{0}$ is suddenly applied to the crack 


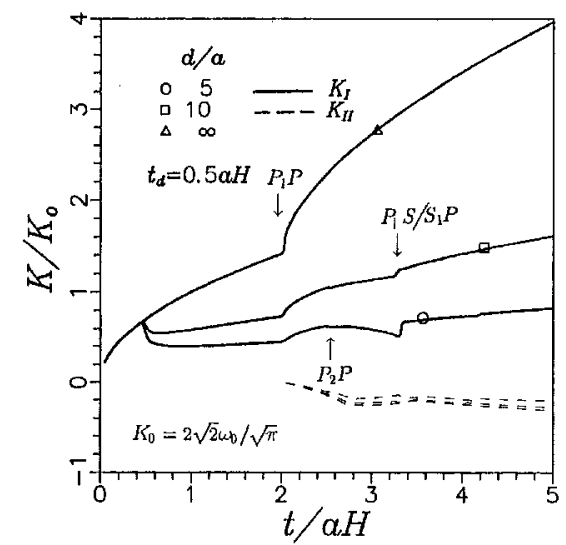

Fig. 3 Stress intensity factors of a propagating crack subjected to a uniform pressure. The half-plane boundary is parallel to the crack.

faces. The pressure continues to be applied along the newly extending crack faces $-v t_{d}<x_{1}<0$; after the crack tip pass through the point $x_{1}=0$, the pressure is only applied at the crack faces for $x_{1}<0$ and does not cover the newly created crack faces. The uniform pressure can be expressed as

$$
\bar{\sigma}_{22}=\frac{\sigma_{0}}{2 \pi i} \int \frac{1}{p \lambda(1-\lambda v)} e^{p \lambda\left(\xi-v t_{t}\right)} d \lambda
$$

The emitted stress fields of the propagating crack can be obtained by superimposing the fundamental solution (A14) as follows:

$$
\begin{aligned}
\bar{\sigma}_{22} & =\frac{1}{2 \pi i p} \int \frac{\left(b^{2}(1-\lambda v)^{2}-2 \lambda^{2}\right)^{2}}{\kappa d^{2} \alpha_{+}(1-\lambda v)^{2}\left(c_{1}-\lambda\right) S_{-}(\lambda)} F_{1}(\lambda) e^{-p \alpha x_{2}+p \lambda \xi} \\
& +\frac{4 \lambda^{2} \alpha_{-}(\lambda) \beta}{\kappa d^{2}(1-\lambda v)^{2}\left(c_{1}-\lambda\right) S_{-}(\lambda)} F_{1}(\lambda) e^{-p \beta x_{2}+p \lambda \xi} d \lambda, \quad \text { (19) } \\
\bar{\sigma}_{12} & =\frac{1}{2 \pi i p} \int \frac{-2 \lambda \alpha_{-}(\lambda)\left(b^{2}(1-\lambda v)^{2}-2 \lambda^{2}\right)}{\kappa d^{2}(1-\lambda v)^{2}\left(c_{1}-\lambda\right) S_{-}(\lambda)} F_{1}(\lambda) e^{-p \alpha x_{2}+p \lambda \xi} \\
& +\frac{2 \lambda \alpha_{-}(\lambda)\left(b^{2}(1-\lambda v)^{2}-2 \lambda^{2}\right)}{\kappa d^{2}(1-\lambda v)^{2}\left(c_{1}-\lambda\right) S_{-}(\lambda)} F_{1}(\lambda) e^{-p \beta x_{2}+p \lambda \xi} d \lambda, \quad(20)
\end{aligned}
$$

Table 1 Arrival times at which reflected waves reach the moving crack tip. The half-plane boundary is parallel to the crack.

\begin{tabular}{c|ccc}
$v$ & $P_{1} P\left(P_{2} P\right)$ & $P_{1} S\left(P_{2} S\right) / S_{1} P\left(S_{2} P\right)$ & $S_{1} S\left(S_{2} S\right)$ \\
\hline $0 v_{l}$ & $2.00(2.50) a H$ & $2.73(3.23) a H$ & $3.46(3.95) a H$ \\
$0.1 v_{l}$ & $2.01(2.51) a H$ & $2.75(3.26) a H$ & $3.50(4.02) a H$ \\
$0.2 v_{l}$ & $2.02(2.54) a H$ & $2.80(3.33) a H$ & $3.63(4.19) a H$
\end{tabular}

where

$$
F_{1}(\lambda)=\frac{\sigma_{0} \alpha_{+}(0)}{c_{2} S_{+}^{0}(0) \lambda}+\frac{\sigma_{0} \alpha_{+}(d) e^{-p_{d}}}{\left(c_{2}+d\right) S_{+}(d)(d-\lambda)} .
$$

The stress intensity factor can also be obtained by superimposing the fundamental solution (A16) as follows:

$$
\begin{aligned}
\bar{K}_{I}= & \frac{\sigma_{0} \sqrt{2}}{2 \pi i p^{2 / 3}} \\
& \times \int \frac{\alpha_{+}(\lambda)}{\sqrt{1-a v}\left(c_{2}+\lambda\right) S_{+}(\lambda) \lambda(1-\lambda v)} e^{-p \lambda i t} d \lambda .
\end{aligned}
$$

Because only the poles $\lambda=0$ and $d$ contribute to the integral in (22), the stress intensity factor for the unbounded medium in the time domain is

$$
\begin{aligned}
K_{I}=\frac{2 \sqrt{2} \alpha_{+}(d)}{\sqrt{\pi} \sqrt{1-a v}\left(c_{2}+d\right)} & \\
& S_{+}(d) \\
& \times\left(\omega_{0} \sqrt{t}-\sqrt{t-t_{d}} H\left(t-t_{d}\right)\right) .
\end{aligned}
$$

2.2.2 Stress Intensity Factors due to Reflected Waves From the Boundary Parallel to the Crack. The incident and diffracted waves emitted from the propagating crack will be reflected from the horizontal boundary at $x_{2}=H$. From Eqs. (19) and (20), it is obvious that the tractions which must be applied at $x_{2}=H$ to eliminate the stresses $\bar{\sigma}_{22}^{D}$ and $\bar{\sigma}_{12}^{D}$ are represented by the exponential function $e^{p \lambda \xi}$. The reflected waves generated from the half-plane boundary can be obtained by superimposing the fundamental solutions (A4), (A5), (A9), and (A10). From a similar procedure to that indicated in the case of stationary crack, the stress intensity factors can be obtained as

$$
\begin{aligned}
\bar{K}_{I}= & \frac{\sqrt{2} A_{0}}{2 \pi i p^{3 / 2}} \int \frac{-\left(b^{2}(1-\lambda v)^{2}-2 \lambda^{2}\right)^{2}\left(\left(b^{2}(1-\lambda v)^{2}-2 \lambda^{2}\right)^{2}-4 \alpha \beta \lambda^{2}\right)}{\sqrt{1-a v R^{2}}} F_{1}(\lambda) e^{-2 p \alpha H} d \lambda \\
& +\frac{\sqrt{2} A_{0}}{2 \pi i p^{3 / 2}} \int \frac{-16 \lambda^{2} \alpha \beta\left(b^{2}(1-\lambda v)^{2}-2 \lambda^{2}\right)^{2}}{\sqrt{1-a v R^{2}}} F_{1}(\lambda) e^{-p \alpha H-p \beta H} d \lambda \\
& +\frac{\sqrt{2} A_{0}}{2 \pi i p^{3 / 2}} \int \frac{4 \alpha \beta \lambda^{2}\left(\left(b^{2}(1-\lambda v)^{2}-2 \lambda^{2}\right)^{2}-4 \alpha \beta \lambda^{2}\right)}{\sqrt{1-a v R^{2}}} F_{1}(\lambda) e^{-2 p \beta H} d \lambda . \\
\bar{K}_{I I}= & \frac{\sqrt{2} A_{0}}{2 \pi i p^{3 / 2}} \int \frac{-2 \lambda \alpha_{-} \beta_{+}\left(\left(b^{2}(1-\lambda v)^{2}-2 \lambda^{2}\right)^{2}-4 \lambda^{2} \alpha \beta\right)}{\sqrt{1-b v R^{2}}} F_{1}(\lambda) e^{-2 p \alpha H} d \lambda \\
& +\frac{\sqrt{2} A_{0}}{2 \pi i p^{3 / 2}} \int \frac{4 \lambda \alpha_{-} \beta_{+}\left(b^{2}(1-\lambda v)^{2}-2 \lambda^{2}\right)\left(\left(b^{2}(1-\lambda v)^{2}-2 \lambda^{2}\right)^{2}-4 \lambda^{2} \alpha \beta\right)}{\sqrt{1-b v R^{2}}} F_{1}(\lambda) e^{-p \alpha H-p \beta H} d \lambda \\
& +\frac{\sqrt{2} A_{0}}{2 \pi i p^{3 / 2}} \int \frac{-2 \lambda \alpha_{-} \beta_{+}\left(\left(b^{2}(1-\lambda v)^{2}-2 \lambda^{2}\right)^{2}\left(\left(b^{2}(1-\lambda v)^{2}-2 \lambda^{2}\right)^{2}-4 \lambda^{2} \alpha \beta\right)\right.}{\sqrt{1-b v R^{2}}} F_{1}(\lambda) e^{-2 p \beta H} d \lambda
\end{aligned}
$$




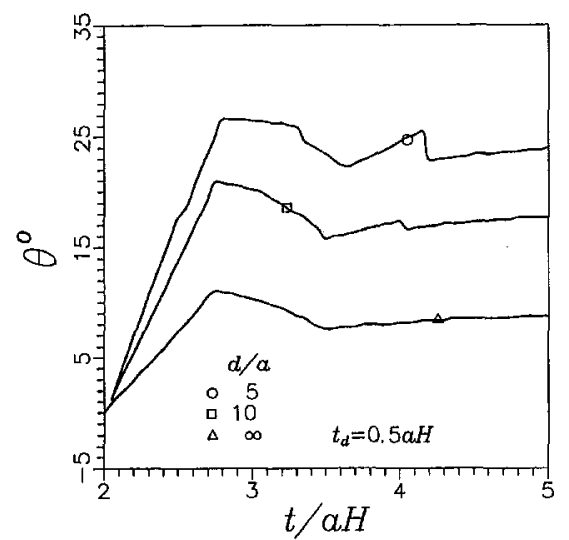

Fig. 4 Possible branching angle $\theta$ of a propagating crack subjected to uniform pressure. The half-plane boundary is parallel to the crack.

By using the Cagniard-de Hoop method, the stress intensity factors can be obtained in the time domain; the expressions are omitted here. The arrival times for $P_{2} P$ and $S_{2} S$ waves are $T_{P_{2} P}=2 \sqrt{a_{1} a_{2}} H$ and $T_{S_{2} s}=2 \sqrt{b_{1} b_{2}} H$, respectively, and $T_{P_{2} S}=$ $T_{S_{2} P}$ is the corresponding time $t$ at which the imaginary part of $\lambda$ begins to vanish and $\alpha(\lambda) H+\beta(\lambda) H=t$ is satisfied.

2.2.3 Stress Intensity Factors due to Reflected Waves From the Boundary Perpendicular to the Crack. In a similar way, the stress intensity factor induced by the reflected waves generated from the vertical boundary can be obtained as follows:

$$
\begin{aligned}
& T_{P_{2} P}=2 a_{1} L, \quad T_{P_{2} S}=\frac{a}{1+b v}+b_{1} L, \\
& T_{S_{2} P}=\frac{b}{1+a v}+a_{1} L, \quad T_{S_{2} S}=2 b_{1} L .
\end{aligned}
$$

\section{Numerical Results}

We now perform a numerical investigation of the dynamic stress intensity factor for a propagating crack interacting with stress waves reflected from boundaries. In this study, Poisson's ratio $\nu$ is assumed to be equal to 0.25 , which gives ratios of the slownesses $b=\sqrt{3} a$ and $c=1.884 a$. A semi-infinite crack embedded parallel to the boundary of a half-plane as shown in Fig. 1 is considered first. At time $t=0$, a uniform loading with magnitude $\sigma_{0}$ is applied to the crack faces. The stress gradually intensifies at the crack tip, and crack propagation may be initiated only after some delay time $t_{d}$. It is assumed that the crack begins to propagate straight with constant speed $v=0,0.1 v_{i}$, $0.2 v_{l}$ after a delay time $t_{d}=0.5 \mathrm{aH}$. The pressure does not expand over the newly created crack faces, but it continues to act on the original crack faces.

The diffracted waves emitted from the crack tip will be reflected from the boundaries and interact with the propagating crack tip. The transient responses of mixed-mode dynamic stress intensity factors are calculated as shown in Fig. 3. The arrival times of reflected waves reaching the propagating crack tip are shown in Table 1. The analytical solutions presented in this study are valid until the $P_{1} P$ wave is reflected twice from the boundary and returns to the moving crack tip. It is found that the mode I stress intensity factors increase rapidly after the

$$
\begin{aligned}
\vec{K}_{I}= & \frac{\sqrt{2}}{2 \pi i p^{3 / 2}} \int \frac{\left(\left(b^{2}-2 a^{2}\right)(1-\tilde{\lambda} v)^{2}+2 \tilde{\lambda}^{2}\right)\left(b^{2}(1-\tilde{\lambda} v)^{2}-2 \tilde{\lambda}^{2}\right) \tilde{\alpha}_{-}\left(b^{2}-2 \bar{\alpha}^{0^{2}}\right)\left(b^{2}-2 \lambda^{2}\right)}{\kappa d^{2}(1-\tilde{\lambda} v)^{2} \tilde{\alpha}^{0}\left(c_{1}-\tilde{\lambda}\right) S_{-}(\tilde{\lambda})(1-\bar{\lambda} v)(1-\lambda v) R^{0}\left(\bar{\alpha}^{0}\right)} F_{2}(\tilde{\lambda}) e^{-2 p \lambda L^{\prime}} \\
& +\frac{4 \tilde{\lambda}\left(b^{2}(1-\tilde{\lambda} v)^{2}-2 \tilde{\lambda}^{2}\right) \tilde{\alpha}-\bar{\alpha}^{0} \beta^{0}\left(\bar{\alpha}^{0}\right)\left(b^{2}-2 \bar{\lambda}^{2}\right)}{\kappa d^{2}(1-\tilde{\lambda} v)^{2}\left(c_{1}-\tilde{\lambda}\right) S_{-}(\tilde{\lambda})(1-\bar{\lambda} v)(1-\lambda v) R^{0}\left(\bar{\alpha}^{0}\right)} F_{2}(\bar{\lambda}) e^{-2 p \lambda L^{\prime}} d \lambda \\
& +\frac{\sqrt{2}}{2 \pi i p^{3 / 2}} \int \frac{-8 \bar{\lambda}^{2} \bar{\beta}^{0^{2}}\left(\left(b^{2}-2 a^{2}\right)(1-\tilde{\lambda} v)^{2}+2 \tilde{\lambda}^{2}\right)\left(b^{2}(1-\tilde{\lambda} v)^{2}-2 \tilde{\lambda}^{2}\right)}{\kappa d^{2}(1-\tilde{\lambda} v)^{2} \tilde{\alpha}_{+}\left(c_{1}-\tilde{\lambda}\right) S_{-}^{0}(\tilde{\lambda})\left(1-\alpha^{0}\left(\bar{\beta}^{0}\right) v\right) R^{0}\left(\bar{\beta}^{0}\right)(1-\lambda v)} F_{2}(\tilde{\lambda}) e^{-p \lambda L^{\prime}-p \alpha^{0}\left(\bar{\beta}^{0}\right)(1-\lambda v) L^{\prime} d \lambda} d \lambda \\
& +\frac{\sqrt{2}}{2 \pi i p^{3 / 2}} \int \frac{8 \tilde{\lambda}^{2} \tilde{\beta} \tilde{\alpha}_{+}\left(b^{2}-2 \beta^{0}\left(\bar{\alpha}^{0}\right)^{2}\right)\left(b^{2}-2 \bar{\lambda}^{2}\right) \bar{\lambda}}{\kappa d^{2}(1-\tilde{\lambda} v)^{2}\left(c_{1}-\tilde{\lambda}\right) S_{-}^{0}(\tilde{\lambda})\left(1-\beta^{0}\left(\bar{\alpha}^{0}\right) v\right) R^{0}\left(\bar{\alpha}^{0}\right)(1-\lambda v)} F_{2}(\tilde{\lambda}) e^{-p \lambda L^{\prime}-p \beta^{0}\left(\bar{\alpha}^{0}\right)(1-\lambda v) L^{\prime}} d \lambda \\
& +\frac{\sqrt{2}}{2 \pi i p^{3 / 2}} \int \frac{16 \tilde{\lambda}^{2} \tilde{\beta}^{0} \tilde{\alpha}-\bar{\beta}^{0^{2}} \alpha^{0}\left(\bar{\beta}^{0}\right) \bar{\lambda}+4 \tilde{\lambda}\left(b^{2}(1-\tilde{\lambda} v)^{2}-2 \tilde{\lambda}^{2}\right) \tilde{\alpha}-\bar{\lambda}^{0} \bar{\beta}^{0}\left(b^{2}-2 \bar{\lambda}^{2}\right)}{\kappa d^{2}(1-\tilde{\lambda} v)^{2}\left(c_{1}-\tilde{\lambda}\right) S_{-}^{0}(\tilde{\lambda})(1-\bar{\lambda} v)(1-\lambda v) R^{0}\left(\bar{\beta}^{0}\right)} F_{2}(\tilde{\lambda}) e^{-2 p \lambda L^{\prime}} d \lambda,
\end{aligned}
$$

where

$$
\begin{gathered}
\tilde{\alpha}=\alpha(\tilde{\lambda}), \quad \tilde{\beta}=\beta(\tilde{\lambda}), \quad L^{\prime}=L+v t_{d}, \\
\bar{\lambda}=\frac{\lambda}{1+\lambda v}, \quad F_{2}(\tilde{\lambda})=\frac{\alpha_{+}(\lambda)}{\sqrt{1-a v}\left(c_{2}+\lambda\right) S_{+}(\lambda)} F_{1}(\tilde{\lambda}) .
\end{gathered}
$$

$\tilde{\lambda}$ expressed in the first and fourth terms in (26) are

$$
\tilde{\lambda}=\frac{-\lambda}{1-2 \lambda v}
$$

and $\tilde{\lambda}$ expressed in the second and third terms in (26) are

$$
\tilde{\lambda}=\frac{-\alpha^{0}\left(\bar{\beta}^{0}\right)}{1-\alpha^{0}\left(\bar{\beta}^{0}\right) v}, \quad \tilde{\lambda}=\frac{-\beta^{0}\left(\bar{\alpha}^{0}\right)}{1-\beta^{0}\left(\bar{\alpha}^{0}\right) v} .
$$

By using the Cagniard-de Hoop method, the stress intensity factors are obtained in the time domain; the results are omitted here. The arrival times are reflected $P_{1} P$ wave reaches the crack tip. The mixed-mode stress intensity factors will lead to crack branching and kinking from the original crack direction. The criterion of minimum strain energy density proposed by Sih (1972) is introduced to predict the crack growth direction. The possible branching angle with respect to the original crack direction is obtained as shown in Fig. 4. It is found that the angles $\theta$ are all positive which means that the crack will propagate upward and extrude out of the half-plane surface.

The diffracted waves generated from the moving crack tip are smaller than those from the stationary crack; at the time that the stress waves generated from the moving crack tip return back to crack tip, the stress intensity factor will experience slight variation. Because the tensile effect of a reflected $P_{2} P$ wave will decay gradually, the incremental rate of stress intensity factor change will decrease after the $P_{2} P$ wave arrives at the crack tip (i.e., about time $t=2.5 a H$ ). But at a later time (about time $t=3.3 a H$ ), the $P_{1} S$ wave which carries a strong tensile effect reaches the crack tip and will enlarge the stress intensity factor. 


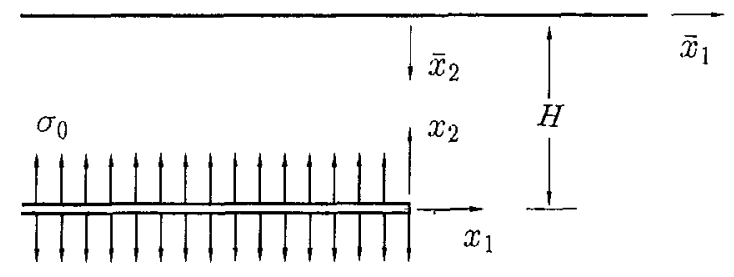

Fig. 5 Configuration and coordinate systems of a semi-infinite crack embedded in a finite strip

If the asymmetrical strip shown in Fig. 5 is considered, and the distance between the crack and the bottom of the strip is $1.4 H$, the mixed-mode dynamic stress intensity factors can be obtained as shown in Fig. 6. The mixed mode stress will make the propagating crack deviate from the original crack direction. The possible kinking angle with respect to the original crack direction is obtained and shown in Fig. 7. The direction of the crack propagation is always toward the top side, which is consistent with the curved path of the propagating crack obtained by Ravi-Chandar and Knauss (1984d).

A semi-infinite crack embedded in a half-plane, as shown in Fig. 2, is considered next. With the same loading condition as the previous problem, the transient history of mode I stress intensity factor induced only from the reflected waves is calculated and shown in Fig. 8. Because the influence of the reflected waves from the boundary perpendicular to the crack on the stress intensity factor is very weak, the transient solutions (4) and (23) are excluded from Fig. 8 in order to emphasize the reflected effect. This figure shows clearly that the influence on the stress intensity factor due to reflected waves from the boundary perpendicular to the crack is much smaller than that from the boundary parallel to the crack. The numerical results are consistent with the experimental results obtained by RaviChandar and Knauss (1984d) in that the influence of reflected waves generated from the frontal surface is very weak. It is worthwhile to note that the first few reflected waves produce a compression effect. The arrival times of the reflected waves are shown in Table 2 . The analytical solutions of this study are valid until the reflected $P_{1} P$ wave is reflected twice from the vertical boundary and returns to the moving crack tip.

Finally, experimental results reported by Ravi-Chandar and Knauss are simulated and compared with the present theoretical

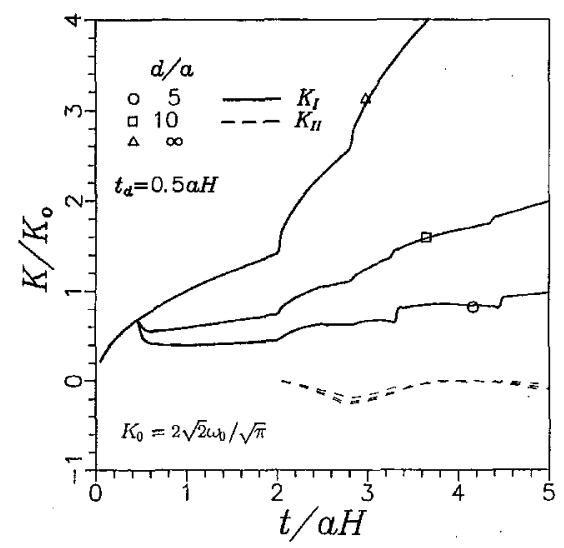

Fig. 6 Stress intensity factors of a propagating crack subjected to uniform pressure in a finite strip

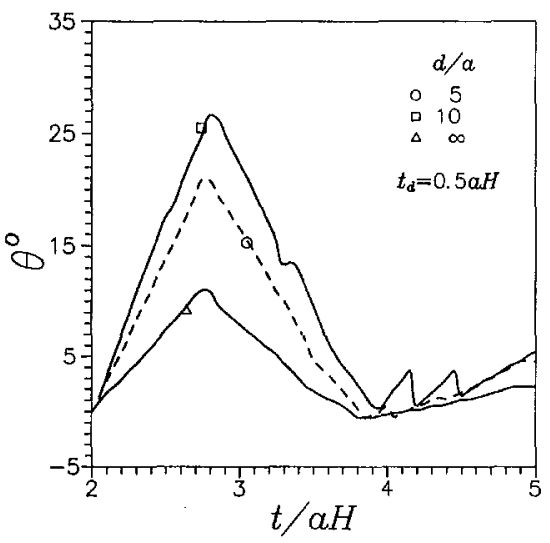

Fig. 7 Possible branching angle $\theta$ of a propagating crack subjected to uniform pressure in a finite strip

analysis. For the experimental set-up, a long edge crack was cut into a rectangular specimen of Homalite- 100 along a symmetrical line as shown in Fig. 9. The specimen was then subjected to suddenly applied uniformly distributed pressure on the crack surfaces. The time variation of this pressure distribution was approximated very well by a linear increment in magnitude up until the rise time $t_{r}$ after application, and then constant magnitude thereafter. From the experimental data presented, the loading rise time $t_{r}$ was approximate $25 \mu \mathrm{s}$, independent of the final magnitude of the applied pressure. The time history of the applied loading can be expressed as $\sigma_{0} t / t_{r} H(t)-\sigma_{0}\left(t-t_{r}\right) /$ $t_{r} H\left(t-t_{r}\right)$, so that the transient effect can be obtained by superposition of the solutions for the linearly increased pressure.

The properties of Homalite- 100 given by Ravi-Chandar and Knauss (1984d) are $\rho=1230 \mathrm{~kg} / \mathrm{m}^{3}, \nu=0.31$, and $E=4550$ $\mathrm{MN} / \mathrm{m}^{2}$, which yield the wave speeds $v_{l}=2057 \mathrm{~m} / \mathrm{s}, v_{s}=1176$ $\mathrm{m} / \mathrm{s}$, and $v_{R}=1081 \mathrm{~m} / \mathrm{s}$. Two different loading conditions will be discussed in detail; we choose $\sigma_{0}=1.10 \mathrm{MPa}(2.46 \mathrm{MPa})$ which makes $t_{d}=56 \mu \mathrm{s}(28 \mu \mathrm{s})$ and crack extending speed $v$ $=240 \mathrm{~m} / \mathrm{s}(363 \mathrm{~m} / \mathrm{s})$. The transient stress intensity factors are calculated by neglecting the diffracted waves generated from the corner of the plate and results are shown in Fig. 10. The theoretical predictions are in agreement with experimental results. The first reflected wave generated from the boundary arrives at the propagating crack tip at $t=150 \mu$ s and causes the stress intensity factor to increase very quickly. At about time $t=170 \mu \mathrm{s}$ to $200 \mu \mathrm{s}$, the experiment shows a short duration period of constant value of stress intensity factor. This phenomenon is attributed to the arrival of the reflected $P_{2} P$. But a short

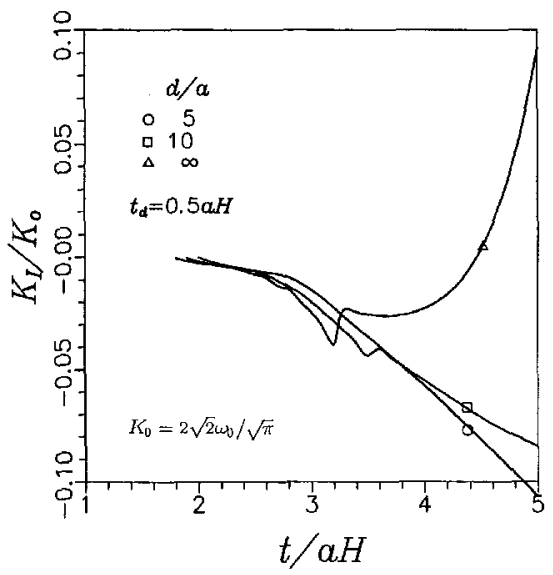

Fig. 8 Stress intensity factors of a propagating crack subjected to uniform pressure. The half-plane boundary is perpendicular to the crack. 
Table 2 Arrival times at which reflected waves reach the moving crack tip. The half-plane boundary is perpendicular to the crack.

\begin{tabular}{c|cccc}
$v$ & $P_{1} P\left(P_{2} P\right)$ & $P_{1} S\left(P_{2} S\right)$ & $S_{1} P\left(S_{2} P\right)$ & $S_{1} S\left(S_{2} S\right)$ \\
\hline $0 v_{l}$ & $2.00(2.50) a L$ & $2.73(3.23) a L$ & $2.73(3.23) a L$ & $3.46(3.95) a L$ \\
$0.1 v_{l}$ & $1.86(2.32) a L$ & $2.40(2.83) a L$ & $2.53(2.98) a L$ & $3.03(3.45) a L$ \\
$0.2 v_{l}$ & $1.75(2.17) a L$ & $2.16(2.53) a L$ & $2.36(2.78) a L$ & $2.70(3.07) a L$
\end{tabular}

time later, the $P_{1} S$ wave and $S_{1} P$ wave reach the crack tip, the stress intensity factor will increase again and branching is observed in the experiment.

\section{Conclusions}

The phenomena of crack propagation, arrest, and branching are the most important subjects in the areas of dynamic fracture analysis. The interaction of reflected waves with a propagating crack subjected to dynamic loading had previously only been investigated in experimental works. It seems difficult to obtain the analytical solutions by using well-known conventional methods. The fundamental solutions for a half-plane and a propagating crack are used to solve the problem of the interaction of stress waves and a moving crack. The reflected and diffracted fields can be constructed by superimposing the fundamental solutions in the Laplace transform domain. A superposition methodology is used in this study to solve this complicated problem. It is shown to be both powerful and efficient in solving complicated dynamic fracture problems of finite dimensions.

In this study, we focus our attention on the contribution of the reflected waves generated from plane boundaries. An explicit result for the dynamic stress intensity factor is obtained in a closed form which is valid before the reflected waves for the second time return to the crack tip. For the finite crack problem, the diffracted waves generated from the other crack tip reach the propagating crack tip will influence the stress intensity factor. But according to the study of a stationary center crack by Sih et al. (1972), the influence is very small before the time $t=2.5 \mathrm{al}$, where $l$ is the crack length. Thus, if the crack length $l=2 H$, the present solutions are remain acceptable during the time interval $t=5 \mathrm{aH}$. Because uniform step loading or high-ramp loading are unusual and the reflected tensile waves occurring require the shorter pulse for the crack close to the surface, the reflected effect due to practical loading should be weaker than the theoretical results.

If the medium is a half-plane or a asymetrical finite strip, the mixed-mode stress intensity factors will induce a curved crack path. The theoretical results and numerical calculations provided in this study are consistent with the experimental work done by Ravi-Chandar and Knauss (1982, 1984d). There are many unanswered questions in dynamic fracture mechanics. This work may provide a useful technique for further investigation in more complicated dynamic loadings and configurations.

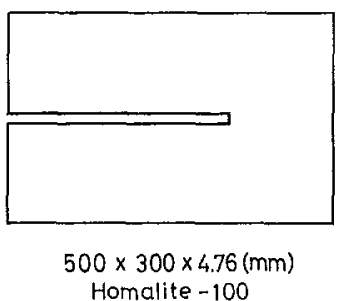

Fig. 9 Configuration of an edge crack specimen investigated by RaviChandar and Knauss $(1982,1984 b)$

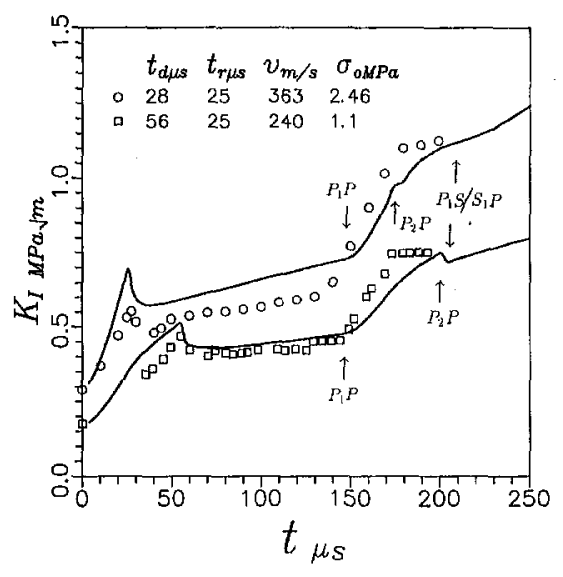

Fig. 10 Stress intensity factors of theoretical prediction and experimental results of Ravi-Chandar and Knauss (1982, 1984b)

\section{Acknowledgment}

The authors gratefully acknowledge the financial support of this research by the National Science Council (Republic of China) under Grant NSC 81-0401-E211-503 to Huafan University.

\section{References}

Freund, L. B., 1972a, "Crack Propagation in an Elastic Solid Subjected to General Loading-I. Constant Rate of Extension," Joumal of the Mechanics and Physics of Solids, Vol. 20, pp. 129-140.

Freund, L. B., 1972b, "Crack Propagation in an Elastic Solid Subjected to General Loading-II. Non-uniform Rate of Extension," Joumal of the Mechanics and Physics of Solids, Vol. 20, pp. 141-152.

Freund, L. B., 1973, "Crack Propagation in an Elastic Solid Subjected to General Loading - III. Stress Wave Loading," Journal of the Mechanics and Physics of Solids, Vol. 21, pp. 47-61.

Freund, L. B., 1974, "Crack Propagation in an Elastic Solid Subjected to General Loading-IV. Obliquely Incident Stress Pulse," Joumal of the Mechanics and Physics of Solids, Vol. 22, pp. 137-147.

Kim, K. S., 1985, "Dynamic Fracture Under Normal Impact Loading of the Crack Faces," ASME Journal, of APPliEd Mechanics, Vol. 52, pp. 585-592.

Ma, C. C., and Chen, S. K., 1994, "Exact Transient Full-Field Analysis of an Antiplane Subsurface Crack Subjected to Dynamic Impact Loading," ASME JoURNAL OF APPLIED MECHANICS, Vol. 61, pp. 649-655.

Ma, C. C., and Ing, Y. S., 1995, "Transient Analysis of Dynamic Crack Propagation with Boundary Effect," ASME JoURNAL OF APPLIED MECHANICS, Vol. 62 , pp. $1029-1038$.

Ravi-Chandar, K., and Knauss, W. G., 1982, "Dynamic Crack-tip Stresses Under Stress Wave Loading-A Comparison of Theory and Experiment," International Journal of Fracture, Vol, 20, pp. 209--222.

Ravi-Chandar, K., and Knauss, W. G., 1984a, "An Experimental Investigation into Dynamic Fracture: I. Crack Initiation and Arrest," International Journal of Fracture, Vol. 25, pp. 247-262.

Ravi-Chandar, K., and Knauss, W. G., 1984b, "An Experimental Investigation into Dynamic Fracture; II. Microstructural Aspects," International Journal of Fracture, Vol. 26, pp. 65-80.

Ravi-Chandar, K., and Knauss, W. G., 1984c, “An Experimental Investigation into Dynamic Fracture: III. On Steady-State Crack Propagation and Crack Branching," International Journal of Fracture, Vol. 26, pp. 141 154.

Ravi-Chandar, K., and Knauss, W. G., 1984d, "An Experimental Investigation into Dynamic Fracture: IV. On the Interaction of Stress Waves With Propagating Cracks," International Journal of Fracture, Vol. 26, pp. 189-200.

Ravichandran, G., and Clifton, R. J., 1989, "Dynamic Fracture Under Plane Wave Loading,' International Journal of Fracture, Vol. 40, pp. 157-201

Sih, G. C., Embley, G. T., and Ravera, R. S., 1972, "Impact Response of a Finite Crack in Plane Extension," International Journal of Solids and Structures, Vol. 8, pp. 977-993.

Thau, S. A., and Lu, T. H., 1971, "Transient Stress Intensity Factors for a Finite Crack in an Elastic Solid Cause by a Dilatational Wave," International Journal of Solids and Structures, Vol. 7, pp. 731-750.

Tsai, C. H., and Ma, C. C., 1991, "Exact Transient Solutions of Buried Dynamic Point Forces and Displacement Jumps for an Elastic Half Space," International Journal of Solids and Structures, Vol. 28, pp, 955-974.

Tsai, C. H., and Ma, C. C., 1992, "Transient Analysis of a Semi-infinite Crack Subjected to Dynamic Concentrated Forces," ASME Journal of APPLIED MECHANICS, Vol. 59, pp. 804-811.

Tsai, C. H., and Ma, C. C., 1997, "Transient Analysis of a Propagating InPlane Crack in a Finite Geometry body Subjected to Static Loadings," ASME JoURnAL OF APPLIED MECHANICs, accepted for publication. 


\section{A P P E N D I X}

\section{A1 Fundamental Solution of a Half-Plane}

The solution of applying an exponentially distributed loading in the Laplace transform domain to the surface of a half-plane is denoted as the fundamental solution of the half-plane. The coordinates system $\left(\xi, x_{2}\right)$ moving with a constant speed $v$ is attached at the surface of half-plane. For the boundary conditions

$$
\begin{gathered}
\bar{\sigma}_{22}(\xi, 0, p)=e^{p \eta \xi}, \quad-\infty<\xi<\infty, \\
\bar{\sigma}_{12}(\xi, 0, p)=0, \quad-\infty<\xi<\infty,
\end{gathered}
$$

where $\eta$ is an arbitrary imaginary number, the solutions for stresses can be obtained as

$$
\begin{gathered}
\bar{\sigma}_{11}=\frac{\left(\left(b^{2}-2 a^{2}\right)(1-\eta v)^{2}+2 \eta^{2}\right)\left(b^{2}(1-\eta v)^{2}-2 \eta^{2}\right)}{R(\eta)} \\
\times e^{-p \alpha x_{2}+p \eta \xi}+\frac{4 \eta^{2} \alpha \beta}{R(\eta)} e^{-p \beta x_{2}+p \eta \xi}, \quad(A \\
\bar{\sigma}_{12}=\frac{-2 \eta \alpha\left(b^{2}(1-\eta v)^{2}-2 \eta^{2}\right)}{R(\eta)} e^{-p \alpha x_{2}+p \eta \xi} \\
+\frac{2 \eta \alpha\left(b^{2}(1-\eta v)^{2}-2 \eta^{2}\right)}{R(\eta)} e^{-p \beta x_{2}+p \eta \xi}, \quad(A 4) \\
\bar{\sigma}_{22}=\frac{\left(b^{2}(1-\eta v)^{2}-2 \eta^{2}\right)^{2}}{R(\eta)} e^{-p \alpha x_{2}+p \eta \xi} \\
+\frac{4 \alpha \beta \eta^{2}}{R(\eta)} e^{-p \beta x_{2}+p \eta \xi} . \quad(A S)
\end{gathered}
$$

For the boundary conditions

$$
\begin{gathered}
\bar{\sigma}_{22}(\xi, 0, p)=0, \quad-\infty<\xi<\infty, \\
\bar{\sigma}_{12}(\xi, 0, p)=e^{m \xi}, \quad-\infty<\xi<\infty,
\end{gathered}
$$

solutions for stresses are

$$
\begin{array}{r}
\bar{\sigma}_{11}=\frac{-2 \eta \beta\left(\left(b^{2}-2 a^{2}\right)(1-\eta v)^{2}+2 \eta^{2}\right)}{R(\eta)} e^{-p \alpha x_{2}+p \eta \xi} \\
+\frac{-2 \eta \beta\left(\left(b^{2}(1-\eta v)^{2}-2 \eta^{2}\right)\right.}{R(\eta)} e^{-p \beta x_{2}+p \eta \xi} \\
\bar{\sigma}_{12}=\frac{4 \eta^{2} \alpha \beta}{R(\eta)} e^{-p \alpha x_{2}+p \eta \xi} \\
\quad+\frac{\left(b^{2}(1-\eta v)^{2}-2 \eta^{2}\right)^{2}}{R(\eta)} e^{-p \beta x_{2}+p \eta \xi} \\
\bar{\sigma}_{22}=\frac{-2 \eta \beta\left(b^{2}(1-\eta v)^{2}-2 \eta^{2}\right)}{R(\eta)} e^{-p \alpha x_{2}+p \eta \xi} \\
+\frac{2 \eta \beta\left(b^{2}(1-\eta v)^{2}-2 \eta^{2}\right)}{R(\eta)} e^{-p \beta x_{2}+p \eta \xi}
\end{array}
$$

\section{A2 Fundamental Solution of a Propagating Crack}

The solution of an exponentially distributed loading in the Laplace transform domain applied at the propagating crack faces is denoted as the fundamental solution of propagating crack. Consider a semi-infinite crack propagating with constant speed $v=1 / d$ in an unbounded medium. The coordinate system $\left(\xi, x_{2}\right)$ is attached to the moving crack tip and is also moving with the constant speed $v$. Consider the boundary conditions of mode I

$$
\begin{gathered}
\bar{\sigma}_{22}(\xi, 0, p)=e^{p \eta \xi} \text { for } \quad-\infty<\xi<0, \\
\bar{\sigma}_{12}(\xi, 0, p)=0 \text { for }-\infty<\xi<\infty, \\
\bar{u}_{2}(\xi, 0, p)=0 \text { for } \quad 0<\xi<\infty,
\end{gathered}
$$

where $\eta$ is a complex number, under the condition that $\operatorname{Re}(\eta)$ $>\operatorname{Re}(\lambda)$, the stresses can be obtained as follows:

$$
\bar{\sigma}_{i j}=\frac{1}{2 \pi i} \int\left[S_{i j}^{1}(\lambda) e^{-p \alpha x_{2}+p \wedge \xi}+S_{i j}^{2}(\lambda) e^{-p \beta x_{2}+p \lambda \xi}\right] d \lambda,
$$

where

$$
\begin{gathered}
S_{1_{1}}(\lambda)=-\left(\left(b^{2}-2 a^{2}\right)(1-\lambda v)^{2}+2 \lambda^{2}\right) \\
\times\left(b^{2}(1-\lambda v)^{2}-2 \lambda^{2}\right) \alpha_{+}(\eta) G(\eta, \lambda) / \alpha_{+}(\lambda) \\
S_{11}^{2}(\lambda)=4 \lambda^{2} \beta(\lambda) \alpha_{-}(\lambda) \alpha_{+}(\eta) G(\eta, \lambda) \\
S_{1_{2}}(\lambda)=2 \lambda\left(b^{2}(1-\lambda v)^{2}-2 \lambda^{2}\right) \alpha_{-}(\lambda) \alpha_{+}(\eta) G(\eta, \lambda) \\
S_{12}^{2}(\lambda)=-S_{12}^{1}(\lambda) \\
S_{22}^{1}(\lambda)=-\left(b^{2}(1-\lambda v)^{2}-2 \lambda^{2}\right)^{2} \alpha_{+}(\eta) G(\eta, \lambda) / \alpha_{+}(\lambda) \\
S_{22}^{2}=-S_{11}^{2} \\
G(\eta, \lambda)=1 /\left(\kappa(d-\lambda)^{2}\left(c_{1}-\lambda\right)\left(c_{2}+\eta\right)\right. \\
\left.\times S_{-}(\lambda) S_{+}(\eta)(\lambda-\eta)\right)
\end{gathered}
$$

The corresponding mode $I$ stress intensity factor is

$$
\bar{K}_{I}=\frac{-\sqrt{2}}{\sqrt{p}} \frac{\alpha_{+}(\eta)}{\sqrt{1-a v}\left(c_{2}+\eta\right) S_{+}(\eta)} .
$$

If the boundary conditions are

$$
\begin{gathered}
\bar{\sigma}_{22}(\xi, 0, p)=0, \quad-\infty<\xi<\infty, \\
\bar{\sigma}_{12}(\xi, 0, p)=e^{m \xi \xi}, \quad-\infty<\xi<0, \\
\bar{u}_{1}(\xi, 0, p)=0, \quad 0<\xi<\infty,
\end{gathered}
$$

the solution of stresses is expressed as in Eq. (A14) with

$$
\begin{gathered}
S_{11}^{1}(\lambda)=2 \lambda\left(b^{2}(1-\lambda v)^{2}-2 \alpha^{2}\right) \beta_{-}(\lambda) \beta_{+}(\eta) G(\eta, \lambda), \\
S_{11}^{2}(\lambda)=S_{11}^{1}(\lambda), \\
S_{12}^{1}(\lambda)=-4 \lambda^{2} \alpha(\lambda) \beta_{-}(\lambda) \beta_{+}(\eta) G(\eta, \lambda), \\
S_{12}^{2}(\lambda)=-\left(b^{2}(1-\lambda v)^{2}-2 \lambda^{2}\right)^{2} \beta_{+}(\eta) G(\eta, \lambda) / \beta_{+}(\lambda), \\
S_{22}^{1}(\lambda)=2 \lambda\left(b^{2}(1-\lambda v)^{2}-2 \lambda^{2}\right) \beta_{-}(\lambda) \beta_{+}(\eta) G(\eta, \lambda), \\
S_{22}^{2}(\lambda)=-S_{22}^{1}(\lambda) .
\end{gathered}
$$

The corresponding mode II stress intensity factor is

$$
\bar{K}_{I I}=-\frac{\sqrt{2}}{\sqrt{p}} \frac{\beta_{+}(\eta)}{\sqrt{1-b v\left(c_{2}+\eta\right) S_{+}(\eta)}} .
$$

\section{A3 Coordinates Transformation Principle of Stresses}

The physical quantities must be expressed in the same coordinate system, so that the superposition method can be performed successfully. If the stress $\bar{\sigma}_{i j}$ expressed in the fixed coordinate system $\left(x_{1}, x_{2}\right)$ is

$$
\bar{\sigma}_{i j}\left(x_{1}, x_{2}, p\right)=p^{n} \int F(\lambda) e^{-p \alpha^{0} x_{2}+p \lambda x_{1}} d \lambda,
$$

then it can be transformed to the moving coordinate system ( $\xi$, $x_{2}$ ) by the form

$\bar{\sigma}_{i j}\left(\xi, x_{2}, p\right)$

$$
=p^{n} \int(1-\lambda v)^{n-1} F\left(\frac{\lambda}{1-\lambda v}\right) e^{-p \alpha x_{2}+p \lambda \xi} d \lambda,
$$

where $\xi=x_{1}-v t$. The stresses of $(A 22)$ and (A23) can be proved to be equivalent in the time domain. 